\title{
Tamoxifen and tranilast show a synergistic effect against breast cancer in vitro
}

\author{
Darakhshan $\mathrm{S}^{1}$, Ghanbari $\mathrm{A}^{2}$, Gholami Rad F ${ }^{3}$, Bidmeshki Pour A ${ }^{1}$ \\ Department of Biology, Faculty of Science, Razi University, Kermanshah, Iran. a.bidmeshkipour@gmail.com
}

\begin{abstract}
Objective: This study was aimed at examining a separate or combined effect of tamoxifen and tranilast drugs on growth and proliferation of breast cancer cells.

Background: Breast cancer is one of the most common cancers and the second leading cause of cancer death among women worldwide. Tamoxifen is the most widely used anti-estrogen for the treatment of breast cancer. Studies show that a combination therapy with other drugs enhances the activity of tamoxifen. Tranilast is an anti-inflammatory drug. We hypothesize that tranilast plus tamoxifen can work synergistically and help getting better result from this anticancer drug.

Methods: Two breast cancer cell lines, MCF-7 and MDA-MB-231, were treated with graduated concentrations of tamoxifen and tranilast alone or in combination at 24, 48 or 72 hours for MCF-7, and 48 hours for MDA-MB-231 cells. We used the MTT assay and lactate dehydrogenase leakage (LDH) assay to evaluate cell viability and cytotoxicity, respectively.

Results: In both ER-positive and ER-negative breast cancer cell lines, the combination of tranilast and tamoxifen was more effective in growth inhibition than single drug exposure.

Conclusion: We have demonstrated that by means of a synergistic/additive inhibitory effect, tranilast was capable of enhancing the in vitro activity of tamoxifen on breast cancer cell lines. Based on the results obtained in this study, tranilast could be a candidate drug for combination therapy in resistant breast cancer patients (Fig. 9, Ref. 17). Text in PDF www.elis.sk.

Key words: breast cancer, Tamoxifen, Tranilast, LDH release, MTT.
\end{abstract}

Breast cancer is a major cancer leading in both incidence and mortality in women. Worldwide, 1.15 million new cases of breast cancer are diagnosed annually $(1,2)$. Tamoxifen ((Z)-1- $\{4-[2-$ (dimethylamino) ethoxy] phenyl $\}$-1,2-diphenyl-1-butene) (TAM) is a synthetic non-steroidal anti-estrogenic drug widely used for the treatment of breast cancer (3). Throughout the past years, both detection and treatment of breast cancer have evidently improved. Nevertheless, breast cancer has so far remained the main cause of cancer deaths in women. The medical treatment of breast cancer continues to pose a great challenge due to improvement in drug resistance, toxicity and hormonal interplay. Consequently, there is a significant need to investigate new drugs that can be orally administered with diminutive side effects. It is necessary to target breast tumors with various receptor statuses, such as estrogen receptors, and find out whether these new drugs work synergistically with endocrine therapy in order to improve the response rates, better the management of this cancer and potentially extend the survival.

${ }^{1}$ Department of Biology, Faculty of Science, Razi University, Kermanshah, Iran, ${ }^{2}$ Fertility and Infertility Research Center, Kermanshah University of Medical Sciences, Kermanshah, Iran, and ${ }^{3}$ Medical Biology Research Center, Kermanshah University of Medical Sciences, Kermanshah, Iran

Address for correspondence: A. Bidmeshki Pour, Department of Biology, Faculty of Science, Razi University, Kermanshah, Iran. Phone: +988314274545 , Fax: +988314274545

Acknowledgements: The study is supported by Medical Biology Research Center and Fertility and Infertility Research Center, Kermanshah, Iran.
The drug that has been combined with tamoxifen in our study is tranilast, $\mathrm{N}-\left(3^{\prime}, 4^{\prime}\right.$-dimethoxycinnamonyl), anthranilic acid, an anti-allergic agent $(4,5)$ used mainly to treat allergic diseases such as atopic dermatitis and bronchial asthma, presumably by inhibiting the release of chemical mediators from mast cells and basophils (5).

The present study is aimed at investigating the effect of tranilast combined with anti-estrogen therapy based on tamoxifen, in the hope of creating a more effective anti-tumor treatment strategy.

\section{Materials and methods}

\section{Cell culture and Drugs}

MCF-7 (noninvasive human breast cancer cell line, PR and ER-positive) and MDA-MB-231 (invasive and metastatic human breast cancer cell line, PR and ER-negative) obtained from the National Cell Bank, Pasteur Institute of Iran and were maintained in RPMI-1640 culture media supplemented with $10 \%(\mathrm{v} / \mathrm{v})$ fetal calf serum (FCS) and penicillin/streptomycin antibiotics. Cultures were sustained at $37^{\circ} \mathrm{C}$ in a humidified incubator containing $5 \% \mathrm{CO}_{2}$.

Tamoxifen (Enzo Life Sciences) and tranilast (Enzo Life Sciences) were used separately in different concentrations ranging from 1 to $20 \mu \mathrm{M}$ for tamoxifen and from 10 to $200 \mu \mathrm{M}$ for tranilast. The particular concentrations used in alone treatment were the following: 1, 2, 5, 10 and $20 \mu \mathrm{M}$ for tamoxifen, and: 10, 20, 50, 100 and $200 \mu \mathrm{M}$ for tranilast. The synergistic effect of tamoxifen 
with tranilast was examined in 1 concentration of tamoxifen (2 $\mu \mathrm{M})$ with 5 different concentrations of tranilast, namely 10,20 , 50, 100 and $200 \mu \mathrm{M}$.

\section{Lactate dehydrogenase ( $L D H)$ leakage assay}

The LDH release assay is based on the measurement of lactate dehydrogenase activity in the extracellular medium. Reliability, rapidity and easy evaluation are some of the characteristics of this method (6). The activity of LDH in the medium was determined using LDH Cytoxicity Detection Kit (Roche; Germany) which detects LDH enzyme release from damaged or dead cells. Therefore, an increase in LDH activity in each treatment shows that the treatment solution has further dead cells, i.e. it has cytotoxic effects on cells.

Briefly, $1 \times 10^{4}$ cells/well of either MCF-7 or MDA-MB-231 cells was plated in 96-well culture plates for overnight and was allowed to attach for $24 \mathrm{~h}$ before the treatment with drugs. After that, $300 \mu \mathrm{L}$ of fresh media containing all drug concentrations were added to each well (in triplicates) and the plates were incubated at $37{ }^{\circ} \mathrm{C}$ in $5 \% \mathrm{CO}_{2}$. After 24,48 and $72 \mathrm{~h}$ for MCF-7 and $48 \mathrm{~h}$ for MDA-MB-231, the plates were removed from the incubator and then $100 \mu \mathrm{L}$ of media from each well was carefully transferred to new plates. A volume of $100 \mu \mathrm{L}$ of LDH substrate prepared according to the manufacturer's direction was added to each well. After 20-minute shaking at room temperature, the enzymatic reaction was stopped by adding $50 \mu \mathrm{L}$ of $1 \mathrm{M}$ hydrochloric acid. Lactate dehydrogenase activity was determined by a change in absorbance at $490 \mathrm{~nm}$ using an ELISA reader.

\section{MTT assay}

MTT (3-[4,5-dimethylthiazol-2-yl]-2,5-diphenyltetrazolium bromide) is a water soluble tetrazolium salt, which is converted to an insoluble purple formazan. The formazan crystals are impermeable to the cell membranes and therefore they accumulate in viable cells. The MTT assay was tested for its validity in a variety of cell lines (7).

Either MCF-7 or MDA-MB-231 cells were plated in 96-well culture plates at $1 \times 10^{4}$ cells/well density for 24 hours aimed at adherence. All drug concentrations were tested and after incubation for 24, 48 and $72 \mathrm{~h}$ for MCF-7 and $48 \mathrm{~h}$ for MDA-MB-231 at $37{ }^{\circ} \mathrm{C}$ in $5 \% \mathrm{CO}_{2}$, a volume of $20 \mu \mathrm{l}$ of MTT solution (MTT Cell Growth Assay; Merck) (5 mg/ml in PBS) was added to each well and incubated for $4 \mathrm{~h}$ at $37^{\circ} \mathrm{C}$. After near to $4 \mathrm{~h}$ of exposure, the supernatant was eliminated and a volume of $100 \mu$ dimethyl sulfoxide was added to dissolve blue formazan crystals at room temperature for $30 \mathrm{~min}$. The optical density of each well was measured by plate reader at $570 \mathrm{~nm}$. The percentage of cell viability was calculated according to the following equation:

$$
\text { Cell viability }(\%)=\left[\mathrm{A}_{570}(\text { sample }) / \mathrm{A}_{570}(\text { control })\right] \times 100 \%
$$

\section{Statistical analysis}

All assays were performed in triplicate in three independent and separate experiments for both cell lines. Statistical analysis was conducted by using one-way analysis of the variance (ANOVA) and t-test. All statistical analyses were done using SPSS software (version 19.0) and the data were expressed as mean \pm SEM. In all cases, $\mathrm{p}<0.05$ was considered significant.

\section{Results}

In order to evaluate the cytotoxic effects of tamoxifen and/ or tranilast on breast cancer cell lines, the cells were treated with tamoxifen alone (range 1-20 $\mu \mathrm{M}$ ), tranilast alone (range 10-200 $\mu \mathrm{M}$ ), or in combination (examined in $2 \mu \mathrm{M}$ tamoxifen with 5 different concentrations of tranilast: 10, 20, 50, 100 and $200 \mu \mathrm{M}$ ) for 24, 48 and $72 \mathrm{~h}$ in MCF-7 cells and for $48 \mathrm{~h}$ in MDA-MB-231 cells. The percentage of cell survival was assessed by MTT assay and the percentage of cell cytotoxicity was measured by LDH release assay. The results of MTT test are given in Figures 1, 2 and 3 for MCF- 7 and in Figure 4 for MDA-MB-231 cells. The results concerning the LDH assay are given in Figures 5, 6, and 7 for MCF-7 and in Figure 8 for MDA-MB-231 cells.

The percentage of cytotoxicity and the cell viability showed that an appreciable increase in cytotoxic effect was visible in all the cultures treated with the combination of tranilast and tamoxi-

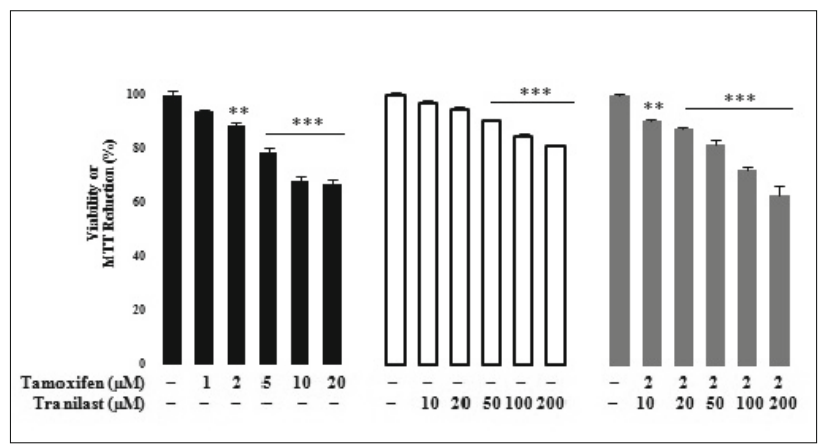

Fig. 1. Bar charts represent the effects of different concentrations of tamoxifen and tranilast as a single or combination $2 \mu \mathrm{M}$ tamoxifen with increasing doses of tranilast on cell viability of MCF-7 cell line at $24 \mathrm{~h}$. Shown are the mean values of three experiments \pm SEM. $p$ values were determined using oneway-ANOVA $\left({ }^{*} p<0.05,{ }^{* *} p<0.01\right.$, $* * * \mathbf{p}<\mathbf{0 . 0 0 1}$ compared with control).

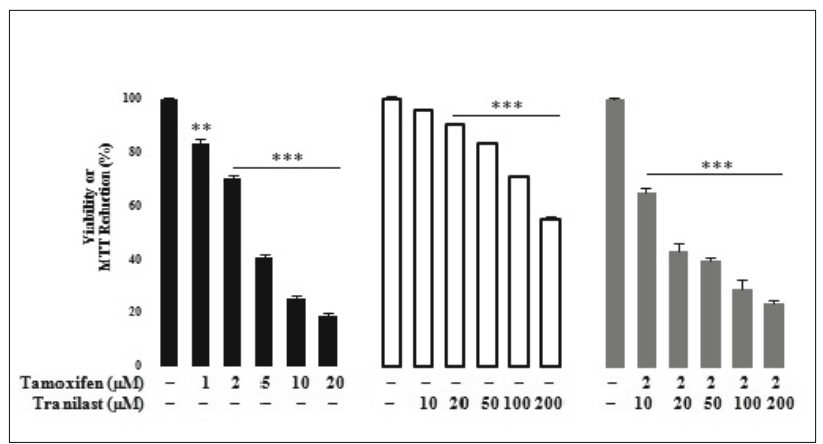

Fig. 2. Bar charts represent the effects of different concentrations of tamoxifen and tranilast as a single or combination $2 \mu \mathrm{M}$ tamoxifen with increasing doses of tranilast on cell viability of MCF-7 cell line at $48 \mathrm{~h}$. Shown are the mean values of three experiments \pm SEM. $p$ values were determined using oneway-ANOVA $\left({ }^{*} p<0.05, * * p<0.01\right.$, $* * * \mathbf{p}<0.001$ compared with control). 


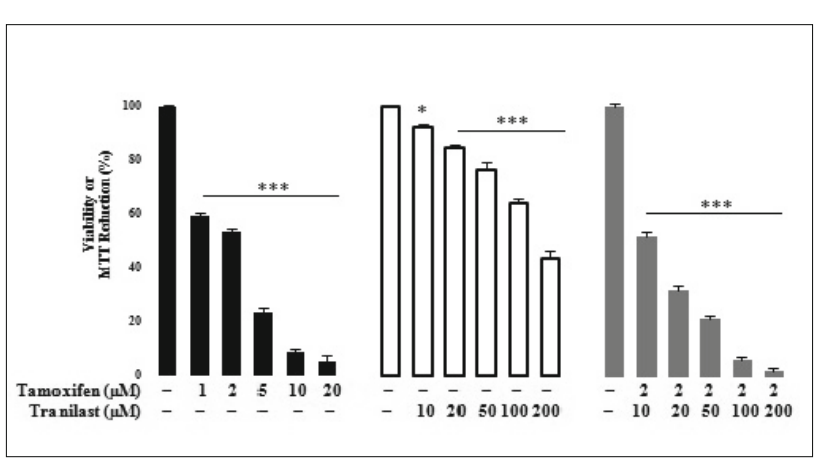

Fig. 3. Bar charts represent the effects of different concentrations of tamoxifen and tranilast as a single or combination $2 \mu \mathrm{M}$ tamoxifen with increasing doses of tranilast on cell viability of MCF-7 cell line at $72 \mathrm{~h}$. Shown are the mean values of three experiments \pm SEM. $p$ values were determined using oneway-ANOVA $(* \mathrm{p}<0.05, * * \mathrm{p}<0.01$, $* * * \mathbf{p}<0.001$ compared with control).

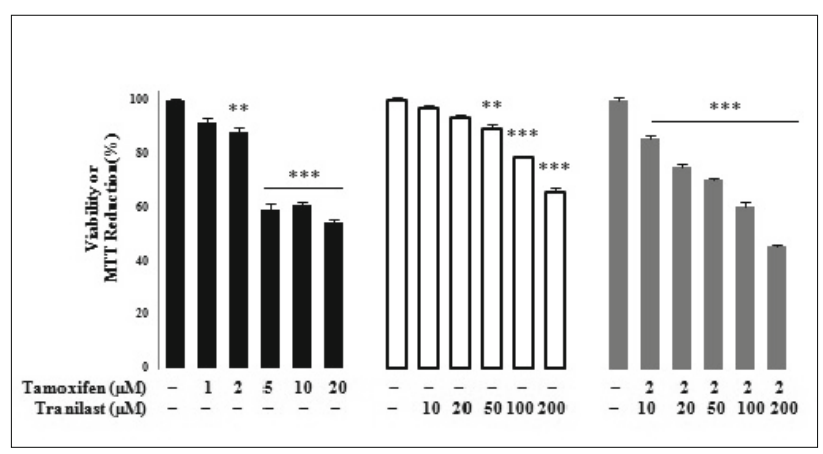

Fig. 4. Bar charts represent the effects of different concentrations of tamoxifen and tranilast as a single or combination $2 \mu \mathrm{M}$ tamoxifen with increasing doses of tranilast on cell viability of MDA-MB-231 cell line at $48 \mathrm{~h}$. Shown are the mean values of three experiments \pm SEM. $p$ values were determined using oneway-ANOVA $(* \mathrm{p}<0.05, * * \mathrm{p}<$ $0.01, * * * \mathrm{p}<0.001$ compared with control).

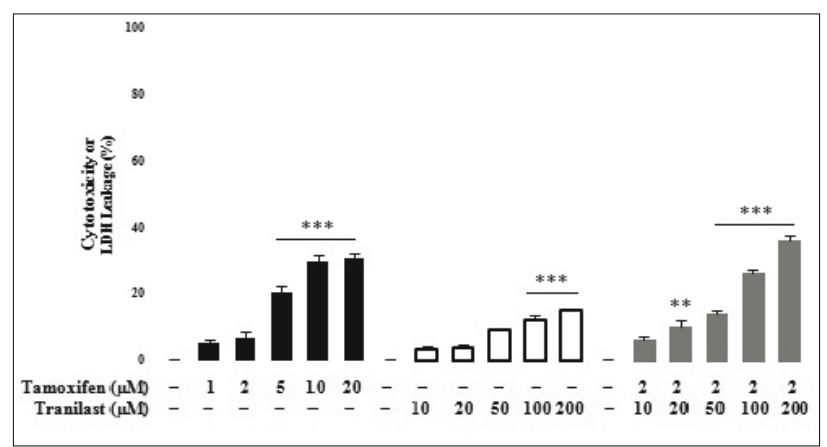

Fig. 5. Cytotoxic effects of different concentrations of tamoxifen and tranilast as a single or combination $2 \mu \mathrm{M}$ tamoxifen with increasing doses of tranilast on MCF-7 cell line at $24 \mathrm{~h}$. Shown are the mean values of three experiments \pm SEM. $p$ values were determined using oneway$\operatorname{ANOVA}(* \mathrm{p}<0.05, * * \mathrm{p}<0.01, * * * \mathrm{p}<0.001$ compared with control).

fen. Cytotoxic effects of tamoxifen as well as tranilast increased in a dose- and time-dependent manner in both MCF-7 and MDAMB-231 cells $(p<0.05)$. Although all doses of tamoxifen and tranilast showed a notable cytotoxic effect, we used a low dose of tamoxifen $(2 \mu \mathrm{M})$ for the combination treatment. After $48 \mathrm{~h}$,

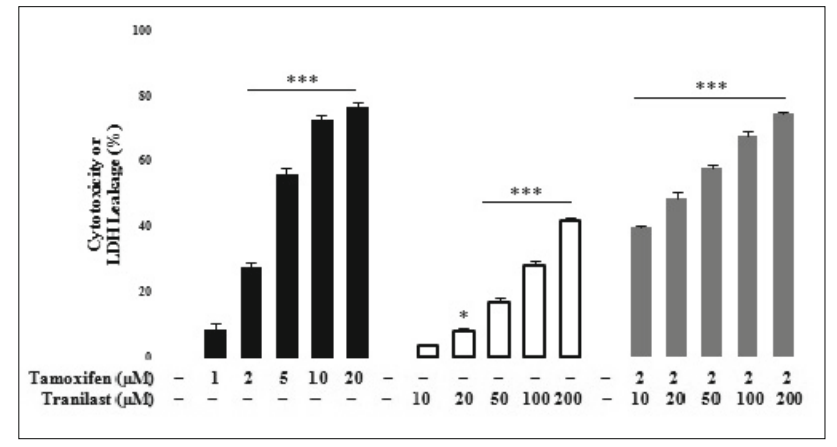

Fig. 6. Cytotoxic effects of different concentrations of tamoxifen and tranilast as a single or combination $2 \mu \mathrm{M}$ tamoxifen with increasing doses of tranilast on MCF-7 cell line at $48 \mathrm{~h}$. Shown are the mean values of three experiments \pm SEM. $p$ values were determined using onewayANOVA (* $p<0.05, * * p<0.01, * * * p<0.001$ compared with control).

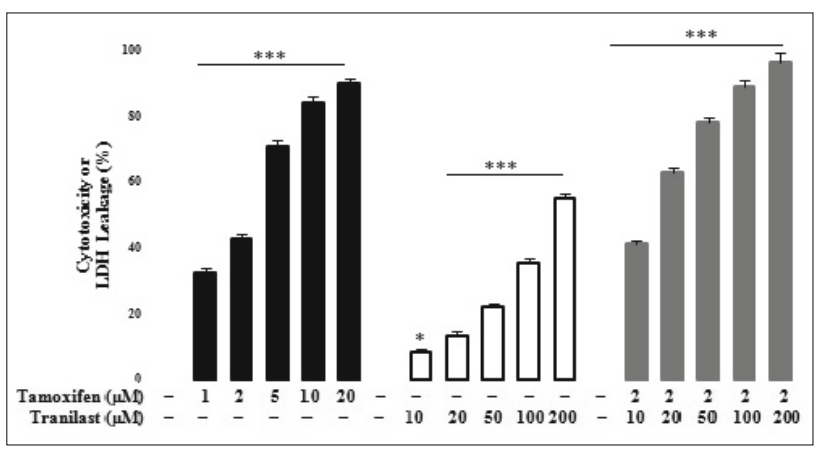

Fig. 7. Cytotoxic effects of different concentrations of tamoxifen and tranilast as a single or combination $2 \mu \mathrm{M}$ tamoxifen with increasing doses of tranilast on MCF-7 cell line at $72 \mathrm{~h}$. Shown are the mean values of three experiments \pm SEM. $p$ values were determined using onewayANOVA $\left({ }^{*} \mathbf{p}<0.05,{ }^{* *} \mathrm{p}<0.01,{ }^{* * *} \mathrm{p}<0.001\right.$ compared with control)

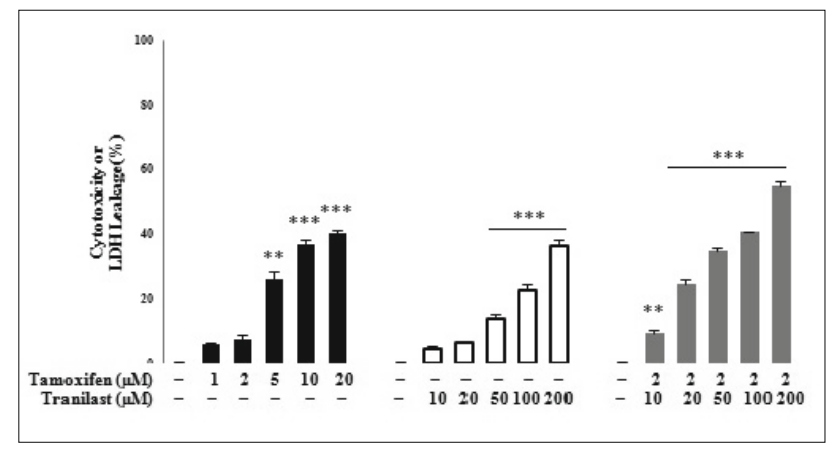

Fig. 8. Cytotoxic effects of different concentrations of tamoxifen and tranilast as a single or combination $2 \mu \mathrm{M}$ tamoxifen with increasing doses of tranilast on MDA-MB-231 cell line at $48 \mathrm{~h}$. Shown are the mean values of three experiments \pm SEM. $p$ values were determined using oneway-ANOVA $\left({ }^{*} p<0.05,{ }^{* *} p<0.01,{ }^{* * *} p<0.001\right.$ compared with control).

$2 \mu \mathrm{M}$ tamoxifen showed $70 \pm 0.8 \%$ of cell viability $(\mathrm{p}<0.001)$, $27 \pm 1.8 \%$ of cytotoxicity $(\mathrm{p}<0.001)$ in MCF-7 cells and $88 \pm$ $1.7 \%$ of cell viability $(\mathrm{p}<0.01), 7 \pm 2.0 \%$ of cytotoxicity $(\mathrm{p}=$ 0.069 ) in MDA-MB-231 cells. Thus, the $2 \mu \mathrm{M}$ dose of tamoxifen was chosen in combination with all concentrations of tranilast for 


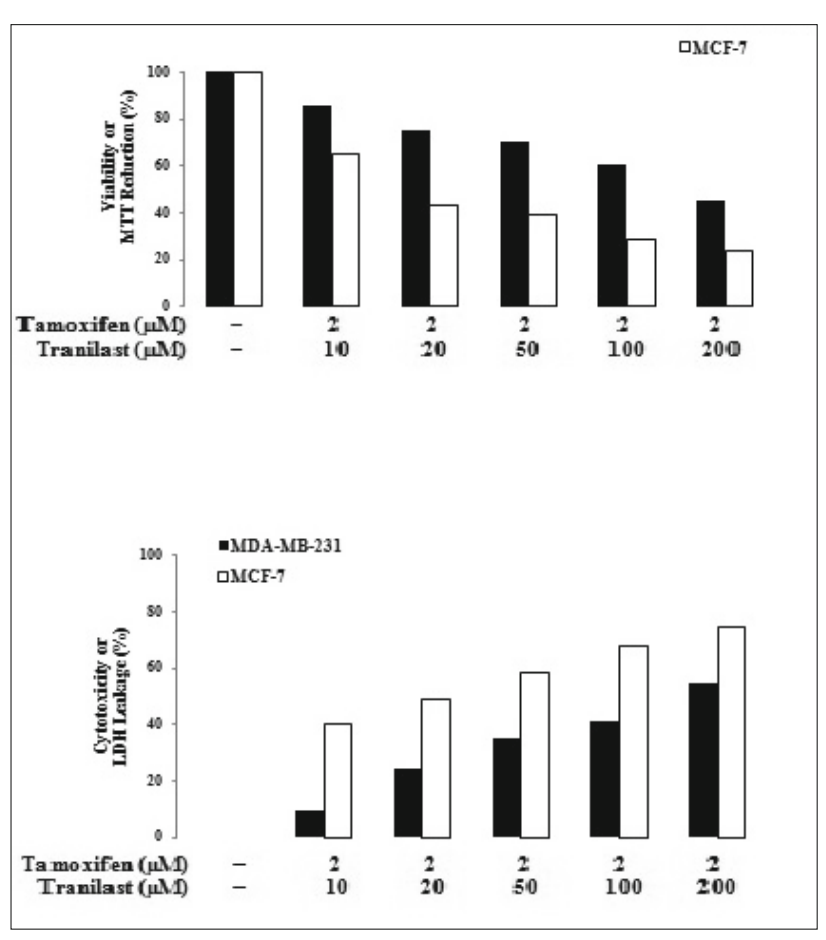

Fig. 9. Comparison of cell viability and cytotoxicity of MCF-7 with MDA-MB-231 following treatment of $2 \mu \mathrm{M}$ tamoxifen with different concentrations of tranilast in combination at $48 \mathrm{~h}$. (A) MTT assay for cell viability; (B) LDH assay for cytotoxicity.

24, 48 and $72 \mathrm{~h}$ in MCF-7 cells and for $48 \mathrm{~h}$ in MDA-MB-231 cells. MCF-7 cells showed additional reduction in cell viability at any concentrations or time points with combination treatment. For the evaluation effects of drugs on MDA-MB-231 cells, the same experiment was also conducted on this cell line and a period of $48 \mathrm{~h}$ was selected for showing any increased therapeutic effect of tamoxifen in combination with tranilast.

The data demonstrated that just as the MCF-7 cells, MDAMB-231 cells had reductions in survival after combined treatment. Nevertheless, there are differences between the two cell lines concerning their sensitivity to drugs and cytotoxic effect of tamoxifen and tranilast on ER-negative breast cancer cell line. The effect of MDA-MB-231 is lower than that of ER-positive breast cancer cell line MCF-7. Surprisingly, all doses of tranilast when combined with $2 \mu \mathrm{M}$ of tamoxifen did decrease the cell viability and of course increased cytotoxicity of MDA-MB-231 cells. Finally, the efficacy of combined treatment was achieved by comparing the data of cell viability and cell cytotoxicity in $48 \mathrm{~h}$ between MCF-7 and MDAMB-231 cell lines (Fig. 9 A, B). There were a few differences in cell viability and cytotoxicity of MCF-7 and MDA-MB-231 when treated with tranilast and tamoxifen in combination.

\section{Discussion}

This study founded that tamoxifen and tranilast suppressed the growth and proliferation of both ER-positive and ER-negative breast cancer cells. The two drugs exhibited significant synergis- tic effects to inhibit the growth of breast cancer even at relatively low concentrations.

Despite an improved understanding, innate or acquired resistance to anti-estrogen therapy remains a significant clinical problem. Although a majority of patients initially benefit from treatment with tamoxifen, $50 \%$ of all metastatic breast cancer patients who receive tamoxifen eventually acquire resistance to tamoxifen (8). The tumor resistance to tamoxifen therapy is based on high dose and long-term usage of the drug and the one of the most prominent aims of studies is to decrease the dose and time of exposure to tamoxifen. Another problem of tamoxifen therapy is due to the existence of ER-negative cells among cell population of tumors.

On the other hand, because endocrine resistance is a major concern in breast cancer recurrence, metastases and survival, numerous laboratories have invested major effort in investigation of new drugs with little side effects that can work synergistically with anti-cancer drugs for better supervision of breast cancer. Preclinical studies suggest that a combination of some drugs or substances and endocrine therapy may help the activity of tamoxifen. This drug is expected to produce better outcomes and stronger anti-tumor effects when compared with endocrine therapy alone.

Recently, a growing attention has been paid to the evaluation of combining the chemotherapeutic drugs with other substances (9-11) for achieving better treatment of breast cancer patients. In this regard, we chose to examine the potential additive effects of tranilast, an anti-allergic drug and tamoxifen, a cancer chemotherapeutic agent for treatment of breast cancer. Tranilast is a drug of low toxicity that is orally administered and studies have clearly shown its antineoplastic effects (12-15). These results of cytotoxicity and viability assays indicate that the combination of tamoxifen and tranilast is synergistic and that the two drugs clearly show a greater effect than any of them alone. Tamoxifen has a low effect on ER-negative breast cancer cells while tranilast increases the efficacy of tamoxifen for ER-negative cells and can also decrease the dose and duration of exposure to tamoxifen in both ER-negative and ER-positive breast cancer cell lines. These results imply that tranilast may be a promising chemosensitizing compound for enhancing the cytotoxic effect of tamoxifen. The anti-tumor effects observed here occurred at concentrations of tranilast that may be well achieved in vivo. Should the results be confirmed in vivo, they may be significant clinically.

It has been shown that inhibition of some breast cancer cell growth by tamoxifen appears to be mediated by TGF-beta (transforming growth factor-beta) signaling pathway (16). One of the major mechanisms that have been elucidated concerning tranilast function is its role in inhibiting the TGF-beta pathway (17). These synergistic anti-proliferative effects were evident in ER-positive and ER-negative breast cancer cells and seem to be independent of ER expression and its pathway. The therapeutic efficacy of tranilast may lie in influencing TGF-beta as a common pathway in both ER-positive and ER-negative breast cancer cells.

In a line, we believe that these data may lead to new therapeutic options for breast cancer patients. 


\section{References}

1. Jemal A, Center MM, Desantis C, Ward EM. Global patterns of cancer incidence and mortality rates and trends. Cancer Epidemiol Biomark Prev 2010; 19: 1893-1907.

2. Jemal A, Siegel R, Ward E, Murray T, Xu J, Thun MJ. Cancer statistics, 2007; CA Cancer J Clin 2007; 57: 43-66.

3. Hoskins JM, Carey LA, McLeod HL. CYP2D6 and tamoxifen: DNA matters in breast cancer. Nat Rev Cancer 2009; 9: 576-586.

4. Azuma H, Banno K, Yoshimura T. Pharmacological properties of $\mathrm{N}-\left(3^{\prime}, 4\right.$ '- dimethoxycinnamonyl) anthranilic acid, a new antiatopic agent. Br J Pharmacol 1976; 58: 483-488.

5. Komatsu H, Kojima M, Tsutsumi N, Hamano S, Kusama H, Ujiie A, Ikeda S, Nakazawa M. Study of the mechanism of inhibitory action of tranilast on chemical mediator release. Jpn J Pharmacol 1988; 46: 43-51.

6. Decker T, Lohmann-Matthes M. A quick and simple method for the quantitation of lactate dehydrogenase release in measurements of cellular cytotoxicity and tumor necrosis factor (TNF) activity. J Immunol Meth 1988; 15: 61-69.

7. Mossmann T. Rapid colorimetric assay for cellular growth and survival: application to proliferation and cytotoxicity assays. J Immunol Meth 1983; 65: 55-63.

8. Shou J, Massarweh S, Osborne CK, Wakeling AE, Ali S, Weiss H, Schiff R. Mechanisms of tamoxifen resistance: increased estrogen receptorHER2/neu cross-talk in ER/HER2- positive breast cancer. J Natl Cancer Inst 2004; 96: 926-935.

9. Rajput S, Kumar BN, Sarkar S et al. Targeted Apoptotic Effects of Thymoquinone and Tamoxifen on XIAP Mediated Akt Regulation in Breast Cancer. Plos One 2013; 8: e61342.
10. Kumar BN, Rajput S, Dey KK, Parekh A, Das S, Mazumdar A, Mandal M. Celecoxib alleviates tamoxifen-instigated angiogenic effects by ROS-dependent VEGF/VEGFR2 autocrine signaling. BMC Cancer 2013; 13: 273 .

11. Sarkar S, Mazumdar A, Dash R, Sarkar D, Fisher PB et al. ZD6474 Enhances Paclitaxel Antiproliferative and Apoptotic Effects in Breast Carcinoma Cells. Journal of Cellular Physiology 2011; 226: 375-384.

12. Platten M, Wild-Bode C, Wick W, Leitlein J, Dichgans J, Weller M. $\mathrm{N}-[3$, 4- dimethoxycinnamoyl]-anthranilic acid (tranilast) inhibits transforming growth factor-beta relesase and reduces migration and invasiveness of human malignant glioma cells. Int J Cancer 2001; 93: 53-61.

13. Sato S, Takahashi S, Asamoto M, Naiki T, Naiki-Ito A, Asai K, Shirai T. Tranilast suppresses prostate cancer growth and osteoclast differentiation in vivo and in vitro. Prostate 2010; 70: 229-38.

14. Yamamoto M, Yamauchi T, Okano K, Takahashi M, Watabe $S$, Yamamoto Y. Tranilast, an anti-allergic drug, down-regulates the growth of cultured neurofibroma cells derived from neurofibromatosis type 1 . Tohoku J Exp Med 2009; 217: 193-201.

15. Subramaniam V, Chakrabarti R, Prud'homme GJ, Jothy S. Tranilast inhibits cell proliferation and migration and promotes apoptosis in murine breast cancer. Anticancer Drugs 2010; 21: 351-361.

16. Buck MB, Pfizenmaier K, Knabbe C. Antiestrogens induce growth inhibition by sequential activation of $\mathrm{p} 38$ mitogen-activated protein kinase and transforming growth factor-beta pathways in human breast cancer cells. Mol Endocrinol 2004; 18: 1643-1657.

17. Prud'homme GJ. Pathobiology of transforming growth factor beta in cancer, fibrosis, and immunologic disease, and therapeutic considerations. Lab Invest 2007; 87: 1077-1091. 